\title{
Influence of Mutual Coupling and Current Distribution Errors on Advanced Phased Antenna Array Nulling Synthesis
}

\author{
Orest G. Vendik ${ }^{1}$, Dmitry S. Kozlov ${ }^{1}$, Michael D. Parnes ${ }^{2}$, Anton I. Zadorozhnyy ${ }^{2}$, Sergey A. Kalinin ${ }^{1}$ \\ ${ }^{1}$ Department of Physical Electronics and Technology, St. Petersburg Electrotechnical University "LETI", St. Petersburg, Russia; \\ ${ }^{2}$ Resonance Ltd., St. Petersburg, Russia. \\ Email: ds_kozlov@list.ru
}

Received October $8^{\text {th }}, 2013$; revised November $7^{\text {th }}, 2013$; accepted December $2^{\text {nd }}, 2013$

Copyright (C) 2013 Orest G. Vendik et al. This is an open access article distributed under the Creative Commons Attribution License, which permits unrestricted use, distribution, and reproduction in any medium, provided the original work is properly cited.

\begin{abstract}
Phased antenna array synthesis procedures based on the amplitude-phase and only-phase distributions providing the side lobe cancellation in a required direction are presented. A receiving antenna array was considered. It is characterized by the direction of the main beam for receiving the useful signal and the additional nulling direction. The required amplitude - phase distribution along the array is provided by the phase shifters and controllable attenuators/amplifiers. Moreover the modified synthesis procedure that takes into account the mutual coupling influence in the antenna array was proposed. Finally the radiation pattern transformation under the influence of the current distribution errors connected with a quality of phase shifters and attenuators/amplifiers was investigated.
\end{abstract}

Keywords: Adaptive Array; Phased Array; Jamming; Array Synthesis

\section{Introduction}

Reconfigurability of a radiation pattern makes it possible to effectively expand phased antenna array applications in communication, radio navigation and radar systems. The most interesting antenna array capability is a shaping and control of a radiation pattern zone with nulling side lobes, which can be used for suppression of jamming. There are many approaches for realization of controllable nulling zone (notch) in the radiation pattern. The first way is using the linear array consisted of two constituents providing the main pattern and the second one centered on the jammer. The signal of the second part is subtracted from the signal of the first one. Hence, the adaptive control sufficiently reduces the gain of the antenna in the direction of the jammer [1-3].

In contrast to that approach, the side lobe cancellation can be provided in a single antenna array by the specially elaborated amplitude and phase distribution. The least mean squares or the sequential quadratic programming algorithms are used as adaptive algorithms to find the required amplitude and phase distribution [4,5].

In order to simplify the adaptive algorithm, the expansion of a radiation pattern into a series of so-called
Sinc-functions was suggested [6-9]. In this paper, we present a nulling procedure of a reconfigurable antenna array due to the amplitude-phase and only-phase distributions. The synthesis is based on the expansion of the desired radiation pattern shape into a series of the Sinc functions.

The mutual coupling in the antenna array can seriously distort the implemented radiation pattern shape. There are different methods for decreasing the mutual coupling effect $[10,11]$. Most of them are complicated and require the design of additional circuits. In contrast to them, the synthesis proposed in this paper allows taking into account the mutual coupling effect during the radiation pattern shaping procedure easily.

In papers devoted to the phased antenna array nulling, there is limited information about the influence of the amplitude-phase errors on the shaping procedure. However the amplitude-phase distortion of the excitation currents can decidedly falsify the results of the synthesis. Thus in this paper this problem was investigated and the criteria for evaluating the acceptable distortion level were considered.

This paper is organized as follows. Section 2 reviews the procedure of the radiation pattern expansion into 
Sinc-functions. In this context, the calculation of currents using found sampling coefficients is discussed. Section 3 then introduces the innovative concept of the current distribution synthesis for the radiation pattern nulling. The influence of mutual coupling in the antenna array is demonstrated in Section 4. The advanced algorithm of the radiation pattern nulling based on only-phase distribution is briefly introduced in Section 5. Then, in Section 6 , an influence of amplitude and phase errors on the notch shaping is presented. This paper concludes with a short summary in Section 7.

\section{Series Expansion of Radiation Pattern into Sinc-Functions}

A starting point of the synthesis procedure is the expansion of the radiation pattern into Sinc-functions which form the orthogonal system [6-9]:

$$
F(u)=\sum_{p=0}^{M-1} N_{p} \cdot \frac{\sin (M \cdot u-\pi \cdot p)}{M \cdot u-\pi \cdot p}
$$

where

$$
u=\frac{\pi d}{\lambda} \sin \theta
$$

Here $M$ is the number of radiators which form the equispaced antenna array, $d$ is the distance between the adjacent radiators, $\lambda$ is the wave length in free space, $N_{p}$ are known as sampling coefficients, $\theta$ is the polar angle.

If the shape of the radiation pattern is determined, in accordance with (1) the system of sampling coefficients $N_{p}$ can be found,

$$
N_{p}=\frac{M}{\pi} \int_{-R}^{R} F(u) \cdot \frac{\sin (M \cdot u-\pi \cdot p)}{M \cdot u-\pi \cdot p} d u .
$$

While the system of the sampling coefficients $N_{\mathrm{p}}$ is known, the excitation current distribution should be found. Take into account that the radiation pattern can be presented in the form (1) or in the ordinary form:

$$
F(u)=\sum_{q=0}^{M-1}\left|I_{q}\right| \cdot e^{j \phi_{q}} \cdot \exp \left[i \cdot 2 u \cdot\left(q-\frac{M-1}{2}\right)\right]
$$

where $\left|I_{q}\right|$ and $\varphi_{q}$ are the amplitudes and phases of the radiator complex currents $I_{q}$.

The number of $I_{q}$ is equal to the number of sampling coefficients $N_{p}$. The radiation patterns in form (1) and in form (4) should be equalized. The equalization forms the system of the linear equations with respect to $I_{q}$ and $N_{p}$. The detailed solution procedure of this equation is presented in Appendix 1. The solution to the system is as follows:

$$
I_{q}=\sum_{p=-\frac{M-1}{2}}^{\frac{M-1}{2}} M_{q, p} N_{p}
$$

where

$$
M_{q, p}=\exp \left[i \pi p\left(1-\frac{1}{M}-\frac{2 q}{M}\right)\right]
$$

\section{Current Distribution Synthesis for the Radiation Pattern Nulling}

In order to demonstrate the radiation pattern shaping with the notch we use the initial excitation current distribution $I_{q}^{(0)}$ (Figure 1) which is used in well known phased antenna arrays:

$$
I_{q}^{(0)}=D+(1-D) \cdot \sin ^{2}\left(\frac{q-\frac{M-1}{2}}{M-1} \pi\right)
$$

where $q$-is the radiator number $(q=0,1,2 \cdots M-1), D$ -is the pedestal height. For all following calculations, we assume that $M=63, D=0.2$.

This current distribution $I_{q}^{(0)}$ forms the radiation pattern $F_{0}$, which can be calculated by (4) (Figure 2).

In order to control the beam-shaping procedure, we use the pattern in the following form:

$$
F_{1}(u)=F_{0}\left(u-u_{0}\right) \cdot Z\left(u, u_{z}, \delta\right)
$$

where

$$
Z\left(u, u_{z}, \delta\right)= \begin{cases}a, & \text { if } u_{z}-\delta<u<u_{z}+\delta \\ 1, & \text { otherwise }\end{cases}
$$

The position of the main beam is determined by the parameter $u_{0}$, the point of the notch in the radiation pattern

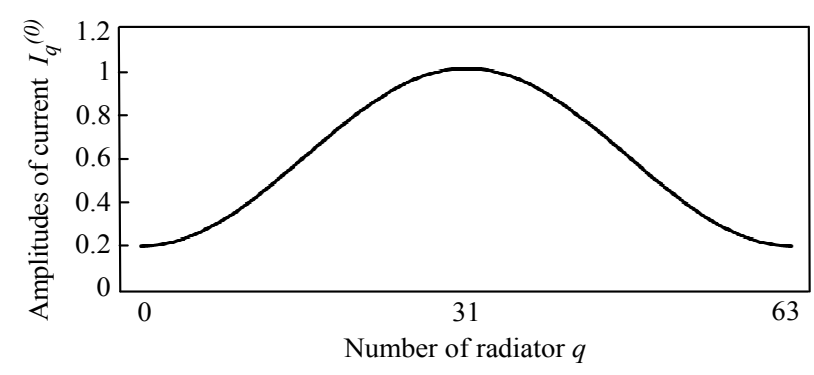

Figure 1. Amplitudes of the excitation currents in the radiators with numbers $q$.

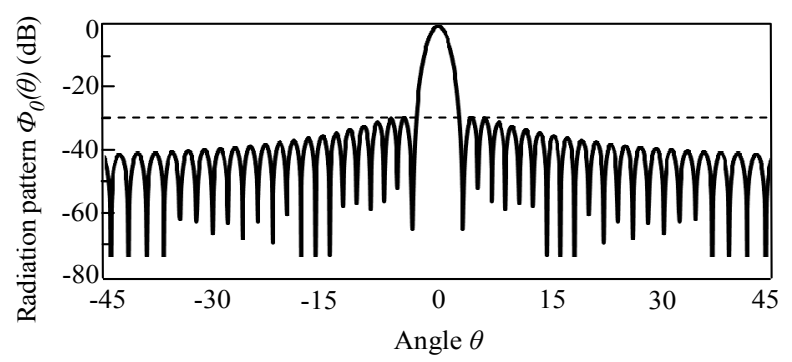

Figure 2. Normalized radiation pattern, presented by the initial excitation current distribution. 
pattern is determined by $u_{z}, \delta$ determines only the width of the notch and does not affect the notch position. The notch depth $\xi$ measured from 0 -dB level is characterized by the parameter $a$. If $a=1$, there is no notch. If $a<0$, the phase of the given pattern is modulated as well. This change of the phase in the given radiation pattern leads to a relevant perturbation of the current distribution along the antenna array.

It is important to stress that the position of the notch and the position of the main beam are independent and determined separately by the parameters $u_{0}$ and $u_{z}$.

The amplitude and phase distribution $\left|I_{q}^{(I)}\right|$ and $\varphi_{q}^{(I)}$ in a linear array can be found using (3) and (5) (Figures 3 and 4).

Substitution the calculated values of the amplitude and phase distribution $\left|I_{q}^{(I)}\right|$ and $\varphi_{q}^{(I)}$ in (4) gives the radiation pattern $\Phi_{1}(\stackrel{\theta}{\theta})$ shown in Figure 5.

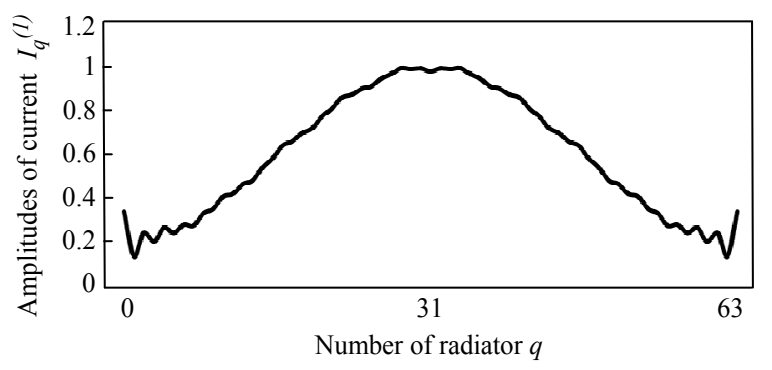

Figure 3. Amplitudes of the currents in the radiators with numbers $q$ for the case of side lobe cancellation in the required direction.

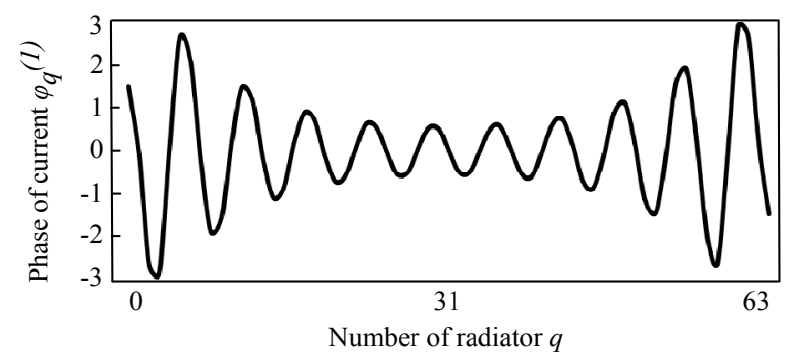

Figure 4. Phases (in degrees) of the currents in the radiators with numbers $q$ for the case of side lobe cancellation in the required direction.

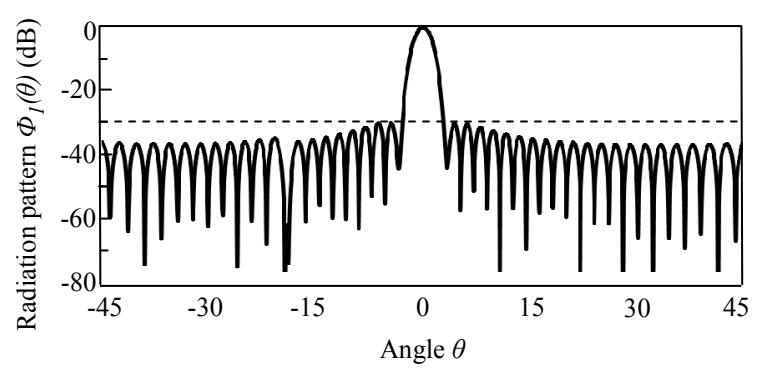

Figure 5. Normalized radiation pattern with the fixed positions of the notch $\theta_{z}$ and the main beam $\theta_{0}\left(\theta_{0}=0^{\circ}, \theta_{z}=-20^{\circ}\right.$; $\delta=2^{\circ}, a=-2, M=63$ ).
The simplified scheme of adaptive antenna array with capability of nulling side lobes using amplitude-phase distribution is shown in Figure 6. It consists of the radiators - 1, the sets of attenuators/amplifiers - 2 and phase shifters - 3 , and the power divider -4 . The power divider determines the initial current distribution. Attenuators/amplifiers and phase shifters are used for adjustment of amplitudes and formation of required phase shifts correspondingly. Both the phase shifters and amplifiers are operating in analog regime providing a corrected linear connection between the bus data impacts and the actual phase and amplitude in the elements of the phased antenna array [12].

\section{Using Influence of the Mutual Coupling in the Antenna Array on the Radiation Pattern Shaping}

The procedure of the radiation pattern shaping presented in the section III was considered for the ideal case when the mutual coupling between radiators in the array is absent. Actually the mutual coupling in the antenna array can be sufficiently strong. In this case the radiation pattern shape can be seriously distorted. That is why the mutual coupling effect should be taken into account during the radiation pattern shaping procedure.

The first step is calculating the impedance matrix $[Z]$ of antenna array $[11,13]$. The mutual coupling between the radiators chosen as half-wave dipoles is described. For simplicity, we consider the coupling between each radiator and its two nearest neighbors, spaced at the distance of $d$ and $2 d$ correspondingly.

As previously described, the divider forms the current distribution $I_{q}^{(0)}$ (7). One can express the mutual coupling relationship as an impedance matrix relating the radiator currents to the applied voltages.

$$
U_{q}^{(0)}=[Z] \cdot I_{q}^{(0)}
$$

where $[Z]$ is the impedance matrix, $U_{q}^{(0)}$ are the applied voltages and $I_{q}^{(0)}$ are the complex amplitudes of the current distribution, as noted above.

The radiation pattern shaped by $U_{q}^{(0)}$ is equivalent to the radiation pattern that formed by the current distribution

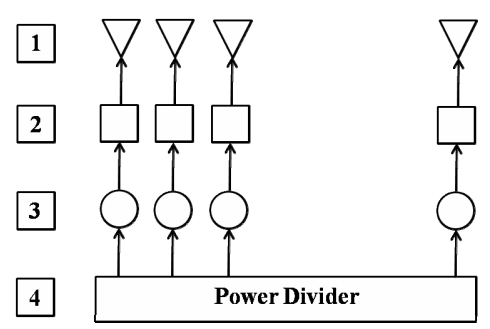

Figure 6. Antenna array controlling system. Drawing symbols: (1) radiators, (2) attenuators/amplifiers of different channels, (3) phase shifters, (4) power divider. 
distribution $I_{q}^{(0 m)}=U_{q}^{(0)} \cdot Y_{11}$, where $Y_{11}$ is the admittance of a single radiator. Thereby, we can say that the presence of coupling between radiators leads to transformation of the current distribution $I_{q}^{(0)}$ into the new one $I_{q}^{(0)}$, which is calculated as:

$$
I_{q}^{(0 m)}=\left([Z] \cdot I_{q}^{(0)}\right) \cdot Y_{11}
$$

Letter " $m$ " in the superscript accounts for the mutual coupling between radiators.

Now we suggest performing the synthesis procedure presented in Section 2 of the paper. After replacing $I_{q}^{(0)}$ by $I_{q}^{(0 m)}$ in all subsequent calculations (1)-(5) one obtains the radiation pattern $\Phi_{2}(\theta)$ (Figure 7). One should compare Figure 2 and Figure 7 realizing that these radiation patterns have been simulated with and without taking into account the mutual coupling between radiators.

The next step refers to the synthesis of current distribution $I_{q}^{(m)}$, which provides the side lobe cancellation in the specified direction. Taking into account the mutual coupling between radiators, one obtains the current distribution $I_{q}^{(1 m)}$, that should be set after the attenuators/ amplifiers and the phase shifters:

$$
I_{q}^{(1 m)}=\left([Z] \cdot I_{q}^{(1)}\right) \cdot Y_{11}
$$

The current distribution $I_{q}^{(1 m)}$ found in this way shapes the radiation pattern $\Phi_{3}(\theta)$ (Figure 8).

After that the transfer coefficients $K_{q}^{(m)}$ (Figure 9) and the phases $\varphi_{q}^{(m)}$ (Figure 10) can be found:

$$
\begin{gathered}
K_{q}^{(m)}=\frac{\left|I_{q}^{(1 m)}\right|}{\left|I_{q}^{(0)}\right|} \\
\varphi_{q}^{(m)}=\arg \left(I_{q}^{(1 m)}\right)
\end{gathered}
$$

The transfer coefficients $K_{q}^{(m)}$ and the phases $\varphi_{q}^{(m)}$ should be used to control the operation of the amplifiers/ attenuators and the phase shifters.

As it is shown, the mutual coupling in the antenna array demands the sufficient correction of the amplitude and phase distribution of the radiator currents. After that

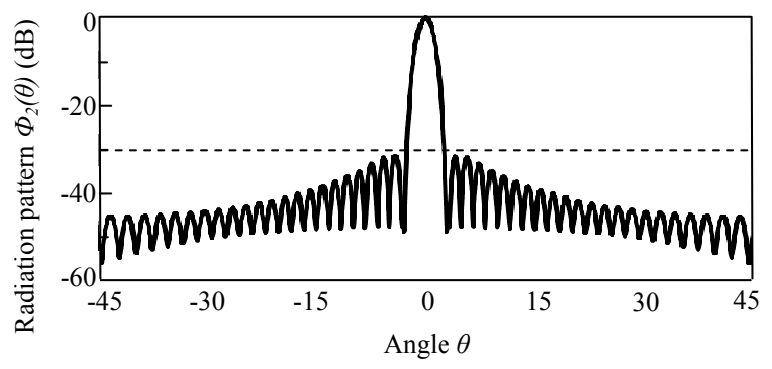

Figure 7. Normalized radiation pattern, presented by the initial excitation current distribution with mutual coupling influence.

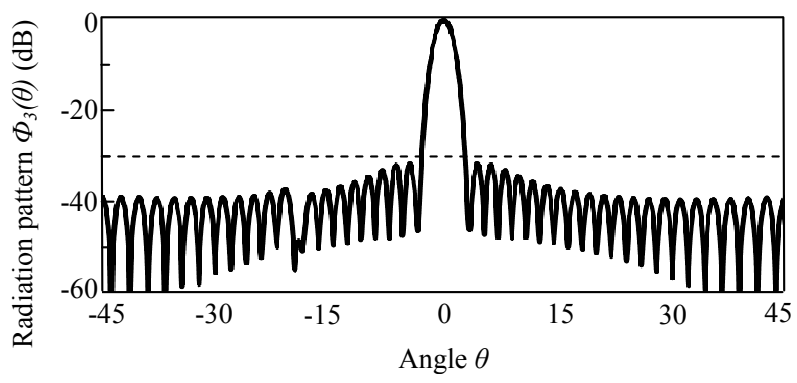

Figure 8. Normalized radiation pattern with mutual coupling influence with the fixed positions of the notch $\theta_{z}$ and the main beam $\theta_{0}\left(\theta_{0}=0^{\circ}, \theta_{z}=-20^{\circ} ; \delta=2^{\circ}, a=-2, M=63\right)$.

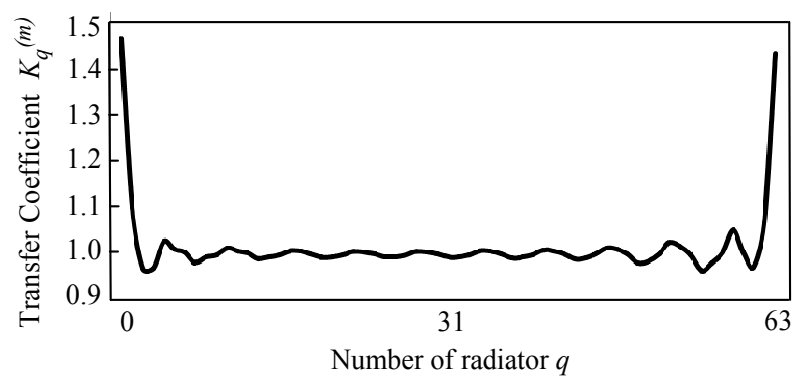

Figure 9. Transfer coefficients of the attenuators/amplifiers for the case of side lobe cancellation in the required direction with mutual coupling influence.

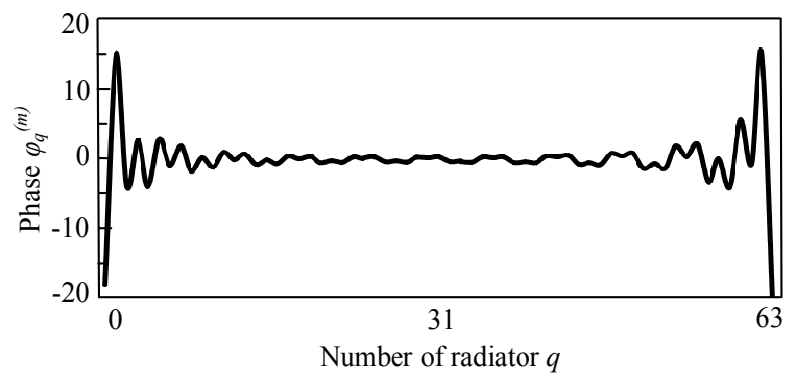

Figure 10. Phases of the phase shifters with numbers $q$ for the case of side lobe cancellation in the required direction.

correction the notch form is weakly affected. Thus, it can be assumed that the proposed radiation pattern shaping procedure with the side lobe cancellation is more or less stable with respect to the mutual coupling effect.

\section{Radiation Pattern Nulling by Only-Phase Distribution}

The shaping synthesis of the radiation pattern with the notch in the required direction was presented in the previous sections of this paper. The proposed procedure requires a modification of the phase and amplitude distribution along the antenna array. This fact leads to using additional attenuators/amplifiers that result in the complication of the whole system. For this reason it was proposed to use the initial amplitude distribution and change only the phase distribution along the array 
according to the described synthesis. Thus the final radiation pattern is determined as:

$$
F_{2}(u)=\sum_{q=0}^{M-1} I_{q}^{(0)} \cdot e^{j \phi_{q}^{(1)}} \cdot \exp \left[i \cdot 2 u \cdot\left(q-\frac{M-1}{2}\right)\right]
$$

where $I_{q}^{(0)}$ - the initial current distribution along the antenna array and $\varphi_{q}^{(I)}$ - the phase distribution obtained by calculations according the synthesis procedure.

The radiation pattern shaped by proposed method is presented in Figure 11. In this case the position and the depth of the notch are not changed, but the additional "upsurge" is appeared.

It should be noted that the correlation between the depth of the notch and the height of the "upsurge" can be varied. In this paper we chose settings to get the sufficiently large depth of the notch. In this case, the height of the "upsurge" is about $6 \mathrm{~dB}$ above the level of the side lobes.

Thus, two shaping procedures: amplitude-phase and only-phase distribution were compared. Amplitude-phase synthesis allows obtaining a more equable radiation pattern without "upsurge". However in this case the radiation pattern control system is more complicate because the additional set of the attenuators/amplifiers is required. If we use this synthesis procedure based on the onlyphase distribution the set of attenuators/amplifiers can be deleted.

\section{Influence of Amplitude and Phase Errors on Formation of Notch in the Radiation Pattern}

The procedure of the antenna array synthesis presented above was considered for the case of the amplitude-phase current distribution along the array without distortion. In reality, the amplitude-phase distribution will be distorted due to various errors.

In this section the distortion of the original radiation pattern under the influence of errors will be considered. The level of errors depends on the quality of the phase shifters, attenuators/amplifiers and power divider.

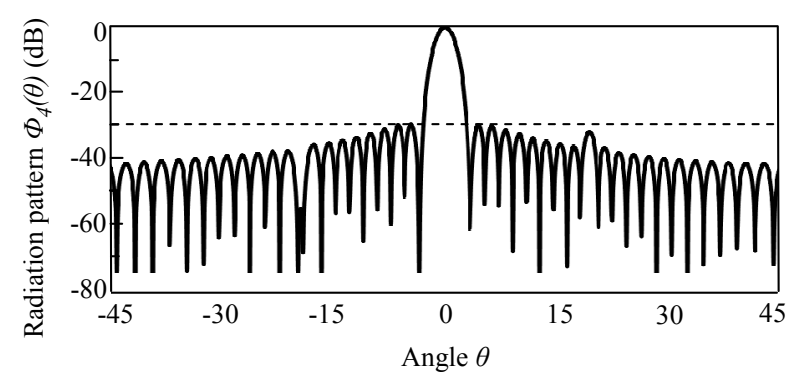

Figure 11. Normalized radiation pattern with the fixed positions of the notch $\theta_{z}$ and the main beam $\theta_{0}$ shaped by only-phase distribution synthesis $\left(\theta_{0}=0^{\circ}, \theta_{z}=-20^{\circ} ; \delta=2^{\circ}, a\right.$ $=-2, M=63)$.
The current distribution is found as a result of synthesis: the amplitude $I_{q}$ and the phase $\varphi_{q}$ do not contain errors. The current and phase distribution with errors can be determined by the following equations:

$$
\begin{gathered}
I_{q \text { error }}=I_{q}+\Delta I_{q} \\
\varphi_{q \text { error }}=\varphi_{q}+\Delta \varphi_{q}
\end{gathered}
$$

where $\Delta I_{q}$ and $\Delta \varphi_{q}$ are the amplitude and phase errors of complex currents of the radiators. The errors $\Delta I_{q}$ и $\Delta \varphi_{q}$ are in the range $\left[-\Delta I_{q \max }, \Delta I_{q \max }\right]$ and $\left[-\Delta \varphi_{q \max }, \Delta \varphi_{q \max }\right]$, where $\Delta I_{q \max }$ and $\Delta \varphi_{q \max }$ are the maximum deviation of $I_{q}$ и $\varphi_{q}$. Values $\Delta I_{q}$ и $\Delta \varphi_{q}$ are random numbers which are allocated within the range determined above. The set of the random numbers was found by using a built-in MathCAD function $\boldsymbol{r n d}(\boldsymbol{x})$ [14].

The selected pattern parameters $\left(\theta_{0}, \theta_{z}, \delta, a, M\right)$ are presented in Figures 5 and 11. The errors $\Delta I_{q}$ and $\Delta \varphi_{q}$ are randomized. Now we should find the statistical characteristics of the notch parameters. Thereto principles of the probability theory [15] should be used.

Let us consider variety of depth of the notch $\xi$ for randomized pairs of errors $\Delta I_{q}$ and $\Delta \varphi_{q}$. For hundred pair of $\Delta I_{q}$ and $\Delta \varphi_{q}$ the values of depth of the notch have been found. The set of the values of depth of the notch is named as a sampling. In the case considered firstly one obtains the random samples $\left(\xi_{1}, \xi_{2} \cdots \xi_{100}\right)$. The next step is formation of nondecreasing sequence $\left(\xi_{1} \leq \xi_{2} \leq \cdots \leq \xi_{100}\right)$, which is characterized by transposed numbers of the sequence. Such a nondecreasing sequence is named as a variation series. The graphical presentation of the variation series as a function of the transposed numbers of the selected pairs $\Delta I_{q}$ and $\Delta \varphi_{q}$ is presented in Figure 12.

The variation series is used to construct the empirical distribution function (see Appendix 2). The empirical distribution function may be named as a probability $P(\xi)$ for obtaining the maximum notch depth $\xi$. The simulation of $P(\xi)$ is worked out for the set of $\left|\Delta \varphi_{q \max }\right|$, which are equal to $0.1^{\circ}, 0.3^{\circ}, 0.5^{\circ}, 1.0^{\circ}, 2.0^{\circ}$, and $4.0^{\circ}$ respectively, for the case of maximum amplitude error $\Delta I_{q \text { max }}=0.2 \mathrm{~dB}$ (Figure 13(a)) and for the for the set of maximum amplitude errors $0.1,0.2,0.3,0.4$,

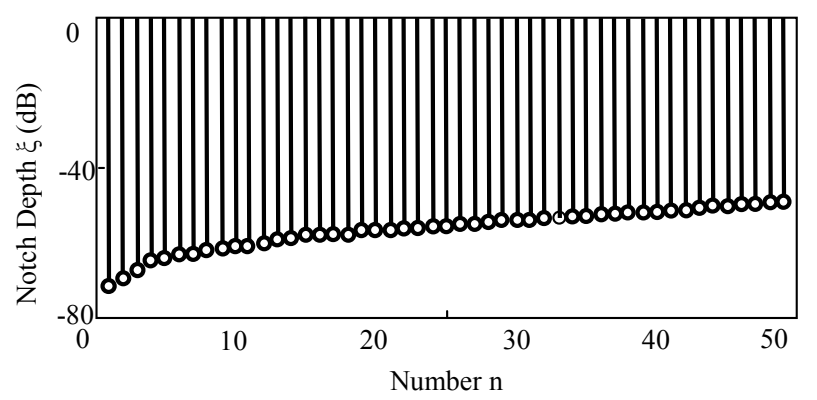

Figure 12. Graphical presentation of variation series. 
$0.5,0.6 \mathrm{~dB}$ in the case of maximum phase error $1.0 \mathrm{de}-$ gree (Figure 13(b)).

The probability $P(\xi)$ as a function of the notch depth was used for a simulation of the expectation value of the notch depth $M\left(\Delta \varphi_{q \max }\right)$, which is presented as a function of the maximum of the phase error $\left|\Delta \varphi_{q \max }\right|$, for $\Delta I_{q \max }=0.2 \mathrm{~dB}$ (Figure 14(a)) and as a function of amplitude errors $\Delta I_{q \max }$ for $\Delta \varphi_{q \max }=1$ degree (Figure 14(b)).

As one can see from Figure 14 the increasing the error is followed by the decreasing the modulus of the notch depth.

The probability $P(\xi)$ as a function of the notch depth was used for a simulation of the dispersion of the notch depth $D\left(\Delta \varphi_{q \max }\right)$, which is presented as a function of the maximum error $\left|\Delta \varphi_{q \max }\right|$, for $\Delta I_{q \max }=$ $\Delta I_{q \max }=0.2 \mathrm{~dB}$ (Figure 15(a)) and and as a function of the maximum amplitude error for $\Delta \varphi_{q \max }=1$ degree (Figure 15(b)).

Dispersion is a measure of deviation of the considered value (the notch depth $\xi$ ) from the expectation value $M\left(\Delta \varphi_{q \max }\right)$. In some approximation, the dispersion can be considered as a width of the notch. The estimation of the notch widths from Figure 15(a) and (b) is about 4 - 5 $\mathrm{dB}$, that is in agreement with notch widths presented in Figures 5 and 11.

The simulation of expectation values and dispersions of notch are explained in Appendix 2.

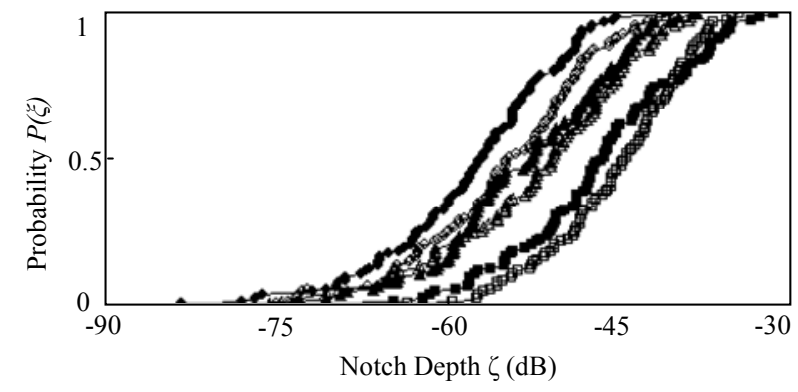

(a)

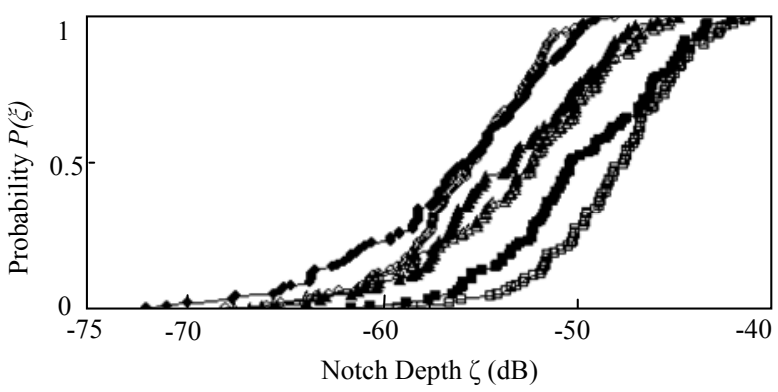

(b)

Figure 13. (a) Probability distribution functions for the maximum amplitude error $0.2 \mathrm{~dB}$ and for maximum phase errors 0.5, 1, 2 degree; (b) Probability distribution functions for the maximum phase error 1 degree for maximum amplitude errors $0.1,0.2,0.4 \mathrm{~dB}$.

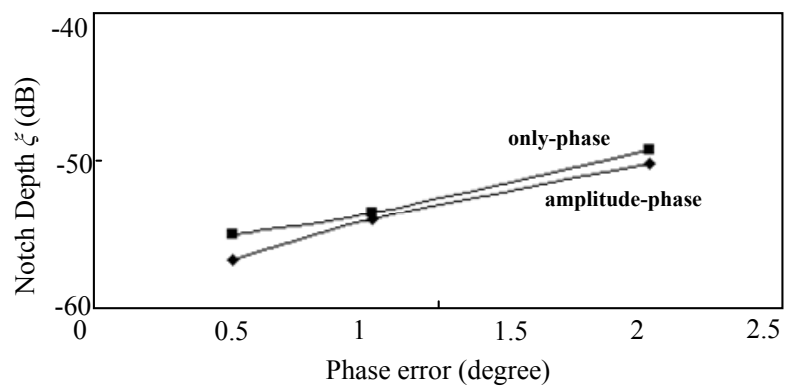

(a)

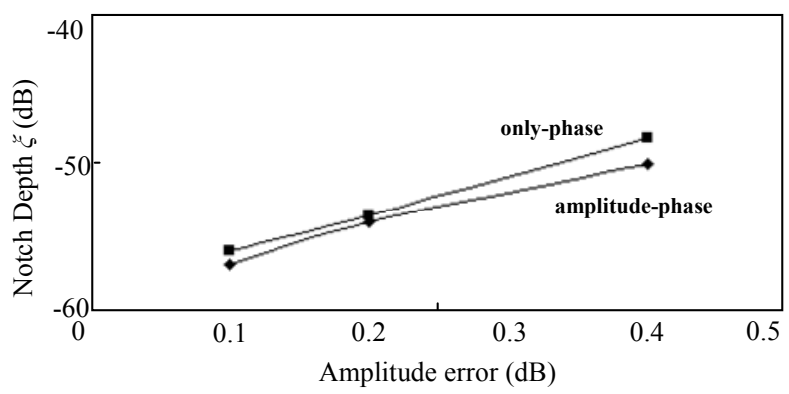

(b)

Figure 14. (a) Expectation value of the notch depth for the maximum amplitude error $0.2 \mathrm{~dB}$ for both types of control; (b) Expectation value of the notch depth for the maximum phase error 1 degree for both types of control.

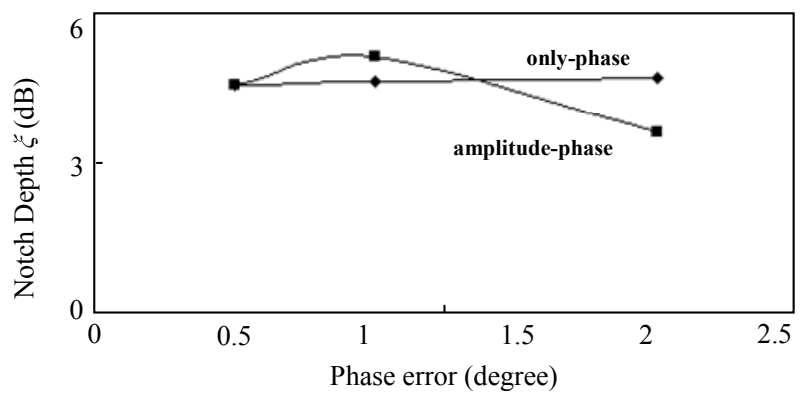

(a)

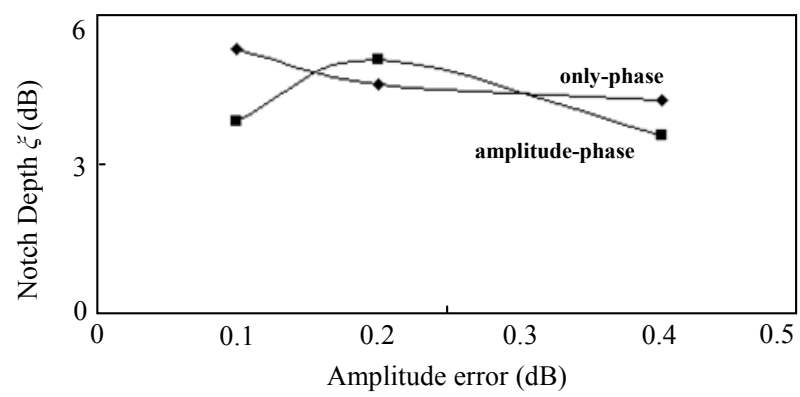

(b)

Figure 15. (a) Dispersion of the notch depth for the maximum amplitude error $0.2 \mathrm{~dB}$ for both types of control; (b) Dispersion of the notch depth for the maximum phase error 1 degree for both types of control.

Application of this analysis for taking into account the error effect on the radiation pattern nulling makes it possible to formulate the requirements for the precision 
of phased antenna array control system.

\section{Conclusion}

The synthesis of an adaptive antenna array has been described. This procedure allows controlling the direction of the main beam for receiving the useful signal and the direction of the notch independently. Practical application of the expansion of a reconfigurable radiation pattern into a series of the Sinc functions has been newly described. This procedure which was additionally modified allowing taking into account the mutual coupling influence in the antenna array was proposed. It was demonstrated that in this case the notch forming does not distort significantly under the mutual coupling influence.

\section{Acknowledgements}

This work was initiated and supported by company "Resonance Ltd.", Saint Petersburg.

\section{REFERENCES}

[1] S. P. Appelbaum, "Adaptive Arrays," IEEE Transactions on Antennas and Propagation, Vol. 24, No. 5, 1976, pp. 585-598. http://dx.doi.org/10.1109/TAP.1976.1141417

[2] R. A. Monzingo and T. W. Miller, "Adaptive Arrays," John Wiley and Sons, New York, 1980.

[3] R. J. Mailloux, "Phased Array Antenna Handbook," Artech House, Boston, London, 1994.

[4] Y. Chu and W.-H. Fang, "A Novel Wavelet-Based Generalized Side Lobe Canceller," IEEE Transactions on Antennas and Propagation, Vol. 47, No. 9, 1999, pp. 14851494. http://dx.doi.org/10.1109/8.793330

[5] M. Mouhamadou and P. Vaudon, "Smart Antenna Array Patterns Synthesis: Null Steering and Multu-User Beam Forming by Phase Control," Progress in Electromagnetics Research, PIER, Vol. 60, 2006, pp. 95-106.
http://dx.doi.org/10.2528/PIER05112801

[6] P. M. Woodward, "A Method of Calculating the Field over a Plane Aperture Required to produce a Given Polar Diagram," Journal I.E.E. (London), Vol. 93, 1947, Part IIIA, pp. 1554-1558.

[7] P. M. Woodward and J. D. Lawson, "The Theoretical Precision with Which an Arbitrary Radiation-Pattern May Be Obtained from a Source of Finite Size," Journal of the Institution of Electrical Engineers-Part III: Radio and Communication Engineering, Vol. 95, No. 37, 1948, pp. 363-370.

[8] O. G. Vendik, "Synthesis of a Line Antenna Array with Non-Mechanical Scanning," Izvestiya Vuzov: Radiotechnika, No. 1, 1960, pp. 77-86. (in Russian)

[9] O. G. Vendik and D. S. Kozlov, "Phased Antenna Array with a Side Lobe Cancellation for Suppression of Jamming," IEEE Antennas and Wireless Propagation Letters, Vol. 11, 2012, pp. 648-650. http://dx.doi.org/10.1109/LAWP.2012.2203780

[10] C. A. Balanis, "Antenna Theory: Analysis and Design," John Wiley \& Sons Inc., New York, 2005.

[11] B. H. Wang and H. T. Hui, "Wideband Mutual Coupling Compensation for Receiving Antenna Arrays Using the System Identification Method," IET Microwaves, Antennas and Propagation, Vol. 5, No. 2, 2011, pp. 184-191. http://dx.doi.org/10.1049/iet-map.2010.0120

[12] A. I. Zadorozhnyy, "C-Band $360^{\circ}$ Digitally-Controlled Analog Phase Shifter with Low Amplitude Modulation Value," Antennas: Radiotechnika, Vol. 10, 2012, pp. 1115. (in Russian)

[13] J. L. Masa-Campos and J. M. Fernadez, "Coupling Characterization and Compensation Model for Antenna Arrays," Journal of Microwave and Optoelectronics, Vol. 4, No. 2, 2005.

[14] www.mathcad.com

[15] D. Stirzaker, "Elementary Probability," Cambridge University Press, Cambridge, 2003. http://dx.doi.org/10.1017/CBO9780511755309 


\section{Appendix 1}

For finding the matrix $M_{q, p}$ the radiation patterns in form (1) and in form (4) should be equalized:

$$
\sum_{q=0}^{M-1} I_{q} \exp \left[i \cdot 2 u \cdot\left(q-\frac{M-1}{2}\right)\right]=\sum_{p=0}^{M-1} N_{p} \cdot \frac{\sin (M \cdot u-\pi \cdot p)}{M \cdot u-\pi \cdot p},
$$

We can multiply the left and right sides of the equation by $\exp [-i \cdot 2 u \cdot(q-(M-1) / 2)]$ and then integrate with respect to $u$ in the range of $-R \pi$ to $R \pi$. ( $R$ is integer) The left part of the equation has the following form:

$$
\int_{-R \pi}^{R \pi} \sum_{q=0}^{M-1} I_{q} \exp \left[i \cdot 2 u \cdot\left(q-\frac{M-1}{2}\right)\right] \cdot \exp \left[-i \cdot 2 u \cdot\left(q-\frac{M-1}{2}\right)\right] d u,
$$

The right part of the equation has the following form:

$$
\sum_{p=0}^{M-1} N_{p} \int_{-R \pi}^{R \pi} \frac{\sin (M \cdot u-\pi \cdot p)}{M \cdot u-\pi \cdot p} \cdot \exp \left[-i \cdot 2 u \cdot\left(q-\frac{M-1}{2}\right)\right] d u,
$$

The functions exp included in the Equation (19) form the orthogonal system:

$$
\int_{-R \pi}^{R \pi} \exp [i \cdot u \cdot l] \cdot \exp [-i \cdot u \cdot m] d u=\left\{\begin{array}{l}
2 \pi R \text { if } l=m \\
0 \text { if } l \neq m
\end{array},\right.
$$

So the left part can be simplified and the initial equation will be transformed:

$$
I_{q}=\sum_{p=0}^{M-1} N_{p} \int_{-R \pi}^{R \pi} \frac{\sin (M \cdot u-\pi \cdot p)}{M \cdot u-\pi \cdot p} \cdot \exp \left[-i \cdot 2 u \cdot\left(q-\frac{M-1}{2}\right)\right] d u,
$$

The main task is integration of the integral in the Equation (22).

The function exp can be expanded by $\sin / \cos$ functions. Thus the required integral is represented as the sum of two simpler integrals:

$$
\begin{aligned}
& \frac{M}{\pi} \int_{-R \pi}^{R \pi} \frac{\sin (M \cdot u-\pi \cdot p)}{M \cdot u-\pi \cdot p} \cdot \exp \left[-i \cdot 2 u\left(q-\frac{M-1}{2}\right)\right] d u \\
& =\frac{M}{\pi} \int_{-R \pi}^{R \pi} \frac{\sin (M \cdot u-\pi \cdot p)}{M \cdot u-\pi \cdot p} \cdot\left[\cos \left[2 u\left(q-\frac{M-1}{2}\right)\right]-i \sin \left[2 u\left(q-\frac{M-1}{2}\right)\right]\right] d u
\end{aligned},
$$

The approximate solutions of these integrals are:

$$
\begin{aligned}
& \frac{M}{\pi} \int_{-R \pi}^{R \pi} \frac{\sin (M \cdot u-\pi \cdot p)}{M \cdot u-\pi \cdot p} \cdot \cos \left[2 u \cdot\left(q-\frac{M-1}{2}\right)\right] d u=\left\{\begin{array}{l}
\cos \left[2\left(q-\frac{M-1}{2}\right) \cdot \frac{\pi \cdot p}{M}\right] \text { if }\left|q-\frac{M-1}{2}\right|<\frac{M}{2}, \\
0 \text { otherwise }
\end{array}\right. \\
& \frac{M}{\pi} \int_{-R \pi}^{R \pi} \frac{\sin (M \cdot u-\pi \cdot p)}{M \cdot u-\pi \cdot p} \cdot \sin \left[2 u \cdot\left(q-\frac{M-1}{2}\right)\right] d u=\left\{\begin{array}{l}
\sin \left[2\left(q-\frac{M-1}{2}\right) \cdot \frac{\pi \cdot p}{M}\right] \text { if }\left|q-\frac{M-1}{2}\right|<\frac{M}{2}, \\
0 \text { otherwise }
\end{array}\right.
\end{aligned}
$$

Finally:

$$
\frac{M}{\pi} \int_{-R \pi}^{R \pi} \frac{\sin (M \cdot u-\pi \cdot p)}{M \cdot u-\pi \cdot p} \cdot \exp \left[-i \cdot 2 u \cdot\left(q-\frac{M-1}{2}\right)\right] d u=\exp \left[-i \cdot 2\left(q-\frac{M-1}{2}\right) \cdot \frac{\pi \cdot p}{M}\right]=\exp \left[i \pi p \cdot\left(1-\frac{1}{M}-\frac{2 q}{M}\right)\right],
$$

The solution accuracy depends on the limits of integration (i.e., the parameter $R$ ) and the number of radiators $M$. It was proposed to estimate the solution accuracy by using the standard deviation $\Delta$ :

$$
\Delta=\sqrt{\frac{\sum_{p} \sum_{q}\left[\left|f_{2}(q, p)-f_{1}(q, p)\right|\right]^{2}}{M}},
$$

where 


$$
\begin{gathered}
f_{1}(q, p)=\frac{M}{\pi} \int_{-R \pi}^{R \pi} \frac{\sin (M \cdot u-\pi \cdot p)}{M \cdot u-\pi \cdot p} \cdot \exp \left[-i \cdot 2 u \cdot\left(q-\frac{M-1}{2}\right)\right] d u \\
f_{2}(q, p)=\left\{\begin{array}{l}
\exp \left[i \pi p \cdot\left(1-\frac{1}{M}-\frac{2 q}{M}\right)\right] \text { if }\left|q-\frac{M-1}{2}\right|<\frac{M}{2} \\
0 \text { otherwise }
\end{array}\right.
\end{gathered}
$$

The simulation results as $\Delta(R, M)$ are given in the table:

\begin{tabular}{|c|c|c|c|c|}
\hline $\mathrm{R}$ & 5 & 15 & 25 & 55 \\
\hline 1 & 0.175 & 0.165 & 0.162 & 0.16 \\
\hline 3 & 0.06 & 0.056 & 0.055 & 0.054 \\
\hline 5 & 0.035 & 0.033 & 0.033 & 0.032 \\
\hline 10 & 0.018 & 0.017 & 0.016 & 0.016 \\
\hline
\end{tabular}

Table 1. Table type styles.

It can be seen that the number of radiators effects not as strong as the integration limits. The deviations are less than $2 \%$ for the value of $R$ more than 10 . Such accuracy is enough.

\section{Appendix 2}

In Section 4 the construction of the variation series was described. The series is used to construct the empirical distribution function:

$$
P(\xi)=\frac{m_{\xi}}{n},
$$

where $m_{\xi}$ is number of terms in series which is smaller than $\xi$. In the case considered $n=100$.

The variation series $\left(\xi_{k}\right)$ can be used for calculation of the sample average, which can be considered as an empirical expectation value of $\xi$ (maximum notch depth) as a function of the maximum deviation of $\Delta I_{\max }$ or $\Delta \varphi_{\max }(\mathrm{see}$ Figure 14):

$$
M\left(\Delta I_{\max }, \Delta \varphi_{\max }\right)=\bar{\xi}=\frac{1}{n} \sum_{k=1}^{n} \xi_{k} .
$$

The variation series $\left(\xi_{k}\right)$ can be used for calculation of the sample standard deviation, which can be considered as a square root from an empirical dispersion for the maximum deviation of $\xi$ ( maximum notch depth) as a function of the maximum deviation $\Delta I_{\max }$ or $\Delta \varphi_{\max }$ (see Figure 15):

$$
D\left(\Delta I_{\max }, \Delta \varphi_{\max }\right)=\frac{1}{n} \sum_{k=1}^{n}\left(\xi_{k}-\bar{\xi}\right)^{2} .
$$

